\title{
A Three-Dimensional Magnetic Force Solution Between Axially-Polarized Permanent-Magnet Cylinders for Different Magnetic Arrangements
}

\author{
Abdel-Karim Daud \\ Electrical Engineering Department \\ Palestine Polytechnic University (PPU) \\ Hebron, Palestine \\ daud@ppu.edu
}

\begin{abstract}
A three-dimensional field solution is presented for axially polarized permanent magnet cylinders. The field components are expressed in terms of finite sums of elementary functions and are easily programmable. They can be used to determine the operating point of rare-earth magnet cylinders. They are also useful for performing rapid parametric calculations of field strength as a function of material properties and dimensions. The field components are developed for different magnet arrangements by taking into account the back iron. Also the method of images is used. Using the field equations, three-dimensional analytical expressions are derived for computing the magnetic force between axiallypolarized permanent-magnet cylinders for different magnetic arrangements. The field calculated results are in good agreement with the experimental data.
\end{abstract}

Keywords-Analytical calculation; magnetic field; permanent magnet; force calculation

\section{INTRODUCTION}

There are numerous devices that use axially polarized permanent magnets (Fig. 1). Examples of these include stepper motors, axial-field permanent-magnet motors, axial couplings and linear PM generators [5-9]. Usually, in these devices, one of the magnets is driven by an external while the other is connected to a load. As the driven magnet moves it impacts a force to the loaded magnet forcing it to move once the loading drag and inertial forces have been overcome. To predict the force, various assumptions can be made that simplify the analysis. In this article, threedimensional analytical expressions are derived for computing the field and the transmitted force. The analysis is based on the assumption of an ideal magnet that is characterized by fixed and uniform polarization. The solution method entails use of the vector potential, and ultimately leads to the numerical integration of the free-space Green's function over two of its spatial variables. Consequently, the resulting field formulae are expressed as discrete sums of elementary functions. One of the key features of this work is that the field formulae can be readily programmed and are ideally suited for rapid parametric studies of field strength at any point outside the magnet [1$3]$. The authors have used these formulae to determine the operating point of existing rare-earth magnets, and to determine the optimum dimensions in order to achieve a specified field profile over an extended spatial region.
The field formulae are developed for different magnet arrangements (Fig. 2) with back iron by using of the method of images [4]. The theory is demonstrated with some sample calculations that are verified with experimental results. Of interest here, for force calculation, only the axial field component of the driven magnet is considered at axial distance equal to $\delta$ from the loaded magnet (Fig. 1). The derived force formulae can be obtained for different magnet arrangements in vertical motion.

They are applicable for magnets made of rare earth materials such as $\mathrm{NdFeB}$.

\section{THEORY}

There are numerous techniques for computing the field due to permanent magnets. We assume that the magnetic material is ideal, uniformly polarized throughout, and then model it as a distribution of equivalent currents. The analysis starts with the magnetostatic field equations for current free regions:

$$
\nabla \times \boldsymbol{H}=0
$$

and

$$
\nabla \times \boldsymbol{B}=0
$$

where $H$ is the magnetic field strength and $B$ is the magnetic flux density. In magnetic materials, the two fields $H$ and $B$ are related to the physical magnetization $M$,

$\boldsymbol{B}=\mu o(\boldsymbol{H}+\boldsymbol{M})$

It is well-known that the two first-order field equations reduce to the second-order equation

$$
\nabla^{2} A=-\nabla \times M
$$

where the vector potential $\boldsymbol{A}$ is given by

$$
\boldsymbol{B}=\boldsymbol{\nabla} \times \boldsymbol{A}
$$

If the magnetization is confined to a volume $V$ and falls abruptly to zero outside, the solution to (4) can be written in the following integral form: 


$$
\boldsymbol{A}(r)=\frac{\mu_{o}}{4 \pi} \int_{V} \frac{J_{M}\left(\boldsymbol{r}^{\prime}\right)}{\left|\boldsymbol{r}-\boldsymbol{r}^{\prime}\right|} d^{3} x^{\prime}+\frac{\mu_{o}}{4 \pi} \int_{S} \frac{j_{M}\left(\boldsymbol{r}^{\prime}\right)}{\left|\boldsymbol{r}-\boldsymbol{r}^{\prime}\right|} d a^{\prime}
$$

where $S$ denotes the surface of the magnet and $\boldsymbol{J}_{M}$ and $\boldsymbol{j}_{M}$ are equivalent volume and surface current densities given by

$J_{M}=\nabla \times \boldsymbol{M} \quad$ (volume current density)

and

$$
\boldsymbol{j}_{M}=\boldsymbol{M} \times \boldsymbol{n} \quad \text { (surface current density) }
$$

respectively [4]. For the problem at hand, it is assumed that the magnetization is in the axial $(\mathrm{z})$ direction,

$M(r)= \pm M \hat{r}$

where the \pm indicates the alternating polarity of adjacent poles. It follows from (7) that the volume current density is zero

$\boldsymbol{J}_{M}=\nabla \times \boldsymbol{M}=0$

and, therefore, (6) reduces to

$$
\boldsymbol{A}(\boldsymbol{r})=\frac{\mu_{o}}{4 \pi} \int_{S} \frac{\boldsymbol{j}_{M}\left(\boldsymbol{r}^{\prime}\right)}{\left|\boldsymbol{r}-\boldsymbol{r}^{\prime}\right|} d a^{\prime}
$$

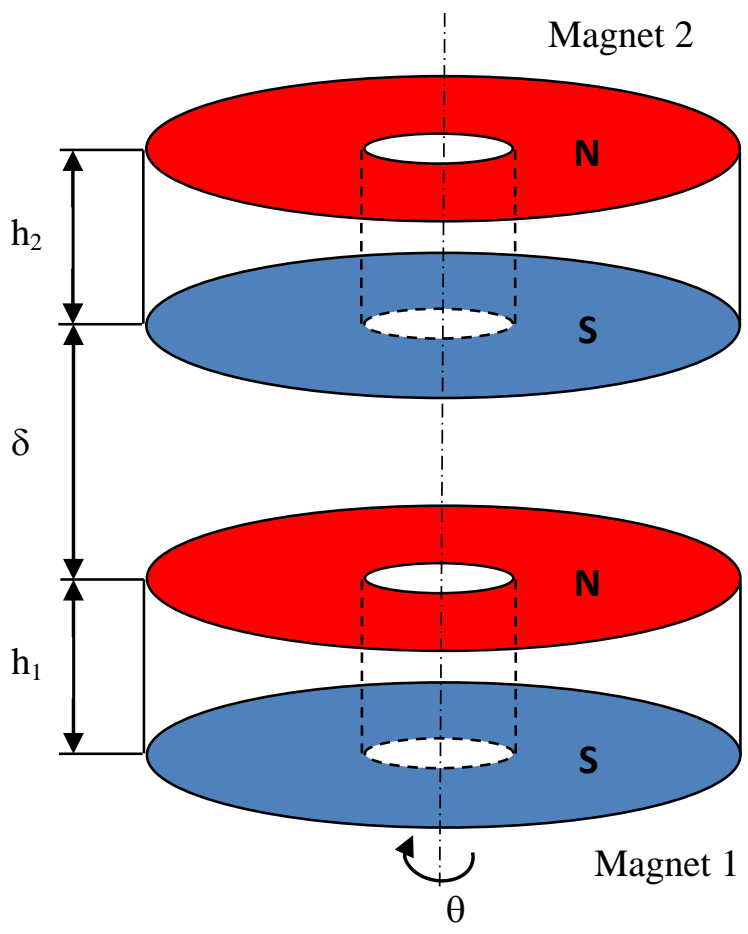

Fig. 1. Two axially polarized cylindrical magnet rings

The $\boldsymbol{B}$ field can be computed from (11) and (5).

First, it is necessary to determine the functional form of $\boldsymbol{j}_{M}$ for the various surfaces as shown in Fig. 2.

From (8) and (9) it follows that $\boldsymbol{j}_{M}=0$ on the top and bottom of the magnet as the magnetization and surface normal are either parallel or antiparallel for these surfaces. There are two remaining surfaces to consider:

$$
\text { inner surface }=\left\{\begin{array}{l}
r^{\prime}=R_{11} \\
0 \leq \theta^{\prime} \leq 2 \pi \\
z^{\prime}(1) \leq z^{\prime} \leq z^{\prime}(2)
\end{array}\right.
$$

outer surface $=\left\{\begin{array}{l}r^{\prime}=R_{12} \\ 0 \leq \theta^{\prime} \leq 2 \pi \\ z^{\prime}(1) \leq z^{\prime} \leq z^{\prime}(2)\end{array}\right.$

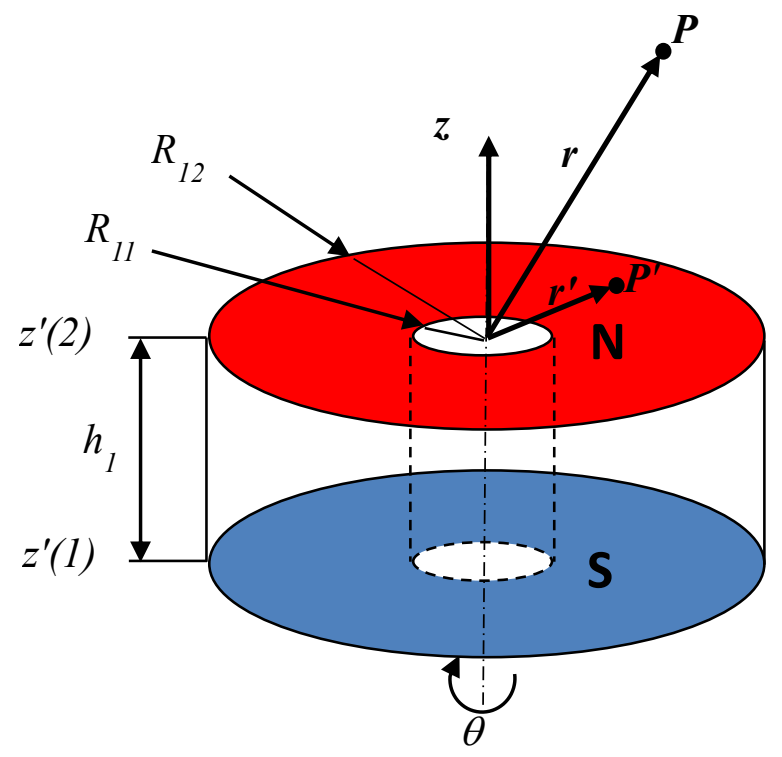

Fig. 2. Axially polarized cylindrical magnet rings

The unit normals for these surfaces are as follows:

$\widehat{\boldsymbol{n}}= \begin{cases}-\hat{\boldsymbol{r}} & \text { (inner surface) } \\ \hat{\boldsymbol{r}} & \text { (outer surface) }\end{cases}$

and, therefore, the corresponding surface current densities are given by

$j_{M}=\left\{\begin{array}{rr}-M \widehat{\boldsymbol{\theta}} & \text { (inner surface) } \\ M \widehat{\boldsymbol{\theta}} & \text { (outer surface) }\end{array}\right.$

Taking into account the results of (12)-(15), (11) can be rewritten as follows

$$
\begin{gathered}
\boldsymbol{A}(\boldsymbol{r})=\left.\frac{\mu_{o} M}{4 \pi} \sum_{j=1}^{2}(-1)^{j} \int_{z^{\prime}(1)}^{z^{\prime}(2)} \int_{0}^{2 \pi} \frac{\widehat{\boldsymbol{\theta}}}{\left|\boldsymbol{r}-\boldsymbol{r}^{\prime}\right|}\right|_{r^{\prime}=r^{\prime}(j)} . \\
\cdot r^{\prime}(j) d \theta^{\prime} d z^{\prime}
\end{gathered}
$$


Note that $\boldsymbol{A}(\boldsymbol{r})$ has no $\mathrm{z}$ component. It can be written in radial component $A_{r}$ and azimuthal component $A_{\theta}$ :

$A_{r}(\boldsymbol{r})=A(r) \cdot \hat{\boldsymbol{r}}$

$A_{\theta}(\boldsymbol{r})=A(r) \cdot \widehat{\boldsymbol{\theta}}$

With

$\hat{\boldsymbol{r}}=\cos (\theta) \widehat{\boldsymbol{x}}+\sin (\theta) \widehat{\boldsymbol{y}}$

and

$\widehat{\boldsymbol{\theta}}=-\sin (\theta) \widehat{\boldsymbol{x}}+\cos (\theta) \widehat{\boldsymbol{y}}$

It follows from (16) that the components $A_{r}$ and $A_{\theta}$ can be written explicity as

$$
\begin{gathered}
A_{r}(\boldsymbol{r})=\left.\frac{\mu_{o} M}{4 \pi} \sum_{j=1}^{2}(-1)^{j} \int_{z^{\prime}(1)}^{z^{\prime}(2)} \int_{0}^{2 \pi} \frac{\sin \left(\theta-\theta^{\prime}\right)}{\left|\boldsymbol{r}-\boldsymbol{r}^{\prime}\right|}\right|_{r^{\prime}=r^{\prime}(j)} . \\
\cdot r^{\prime}(j) d \theta^{\prime} d z^{\prime}
\end{gathered}
$$

and

$$
\begin{gathered}
A_{\theta}(\boldsymbol{r})=\left.\frac{\mu_{o} M}{4 \pi} \sum_{j=1}^{2}(-1)^{j} \int_{z^{\prime}(1)}^{z^{\prime}(2)} \int_{0}^{2 \pi} \frac{\cos \left(\theta-\theta^{\prime}\right)}{\left|\boldsymbol{r}-\boldsymbol{r}^{\prime}\right|}\right|_{r^{\prime}=r^{\prime}(j)} \\
\cdot r^{\prime}(j) d \theta^{\prime} d z^{\prime}
\end{gathered}
$$

Equations (21) and (22) give the vector potential of an axially polarized cylindrical magnet; the field for this magnet $\boldsymbol{B}(\boldsymbol{r})$ can be obtained using (5). Expressions for the three field components: radial component $\left(B_{r}\right)$, azimuthal component $\left(B_{\theta}\right)$, and axial component $\left(B_{z}\right)$ are derived in [10]. For these derivations, it is useful to introduce the Green's function notation

$G\left(\boldsymbol{r} ; \boldsymbol{r}^{\prime}\right)=\frac{1}{\left|\boldsymbol{r}-\boldsymbol{r}^{\prime}\right|}$

which in cylindrical coordinates $(\mathrm{r}, \theta, \mathrm{z})$ reduces to

$G\left(r, \theta, z ; r^{\prime}, \theta^{\prime}, z^{\prime}\right)$
$=\frac{1}{\left[r^{2}+r^{\prime 2}-2 r r^{\prime} \cos \left(\theta-\theta^{\prime}\right)+\left(z-z^{\prime}\right)^{2}\right]^{1 / 2}}$

\section{AXIAL FIELD COMPONENT $\left(B_{z}\right)$}

The axial component of the field follows from (5), specifically,

$B_{z}(\boldsymbol{r})=\frac{1}{r}\left(\frac{\partial}{\partial r}\left(r A_{\theta}(\boldsymbol{r})\right)-\frac{\partial}{\partial \theta} A_{r}(\boldsymbol{r})\right)$

Substitution of (21) and (22) into (25) yields

$$
\begin{aligned}
& B_{z}(\boldsymbol{r})=\frac{\mu_{o} M}{4 \pi} \sum_{j=1}^{2}(-1)^{(j+1)} . \\
& \left.\cdot \int_{z^{\prime}(1)}^{z^{\prime}(2)} \int_{0}^{2 \pi} \frac{r \cos \left(\theta-\theta^{\prime}\right)-r^{\prime}(j)}{\left|\boldsymbol{r}-\boldsymbol{r}^{\prime}\right|^{3}}\right|_{r^{\prime}=r^{\prime}(j)} r^{(j)} d \theta^{\prime} d z^{\prime}
\end{aligned}
$$

The integral in $\theta^{\prime}$ can be evaluated numerically. For this work, Simpson's method was found to be adequate (Appendix). Application of this method to (32) gives an equation for the axial field component,

$$
\begin{aligned}
& B_{z}(\boldsymbol{r})=\frac{\mu_{o} M}{2} \sum_{j=1}^{2}(-1)^{(j+1)} \times \\
& \times\left(\frac{1}{N_{\theta}} \sum_{m=0}^{N_{\theta}} \mathrm{S}_{\theta}(\mathrm{m})\left(r \cos \left(\theta-\theta^{\prime}(m)\right)-r^{\prime}(j)\right) r^{\prime}(j) \times\right. \\
& \left.\times \int_{z^{\prime}(1)}^{z^{\prime}(2)} G^{3}\left(r, \theta, z ; r^{\prime}(j), \theta^{\prime}(m), z^{\prime}\right) d z^{\prime}\right)
\end{aligned}
$$

The remaining integrations in $z^{\prime}$ are evaluated with (50) of the Appendix. Use of this result yields

$$
\begin{gathered}
B_{z}(\boldsymbol{r})=\frac{\mu_{o} M}{2} \sum_{j=1}^{2} \sum_{k=1}^{2}(-1)^{(j+k)} \times \\
\times\left(\frac{1}{N_{\theta}} \sum_{m=0}^{N_{\theta}} \mathrm{S}_{\theta}(\mathrm{m})\left(r \cos \left(\theta-\theta^{\prime}(m)\right)-r^{\prime}(j)\right) r^{\prime}(j) \times\right. \\
\left.\times I_{1}\left(r, \theta, z ; r^{\prime}(j), \theta^{\prime}(m), z^{\prime}(k)\right)\right)
\end{gathered}
$$

where $I_{l}$ is defined in Appendix [10]. Eq. (28) gives the axial field due to the entire magnet.

\section{EFFECT OF BACK IRON}

Let us consider the magnetic circuits shown in Fig. 3. The field in the gap region can be modeled to first order by use of the method of images.

Note that the field can be considered to be a function of the spatial coordinates $(r, \theta, z)$ and the axial position of the magnet, i.e.,

$B_{z}(r, \theta, z)=B_{z}\left(r, \theta, z ; z^{\prime}(1), z^{\prime}(2)\right)$

where $z^{\prime}(1)$ and $z^{\prime}(2)$ are the positions of the bottom and top of the magnet on the $z$ axis, respectively. According to the magnetic circuit shown in Fig. 3a, in which the magnet is in the air gap region, the positions $z^{\prime}(1)$ and $z^{\prime}(2)$ are given in the following equation

$z^{\prime}(1)=-h_{1}, z^{\prime}(2)=0$

With considering the mirror laws of magnetostatic in Cartesian coordinates and under the influence of one-sided back iron according to Fig.3b, eq.(30) can be written as

$z^{\prime}(1)=-2 h_{1}, z^{\prime}(2)=0$ 
While under the influence of double-sided back iron according to Fig.3c, the field components in the air gap can be expressed as a superposition of the fields from an infinite sum of image magnets

$B_{z}(r, \theta, z)=\sum_{i=-\infty}^{+\infty} B_{z}\left(r, \theta, z ; z^{\prime}(1), z^{\prime}(2)\right)$

where the axial positions are given by the following recursive relations:

$z^{\prime}(1)=-2 h_{1}+2 i\left(\delta_{L}+h_{1}\right)$ and

$z^{\prime}(2)=2 i\left(\delta_{L}+h_{1}\right)$

where $\delta_{L}$ is the air gap length between magnet and back iron.

For the magnetic circuits shown in Fig.3a, b and c, the radial positions $r^{\prime}(1)=R_{11}$ and $r^{\prime}(2)=R_{12}$ remain constant.

a)

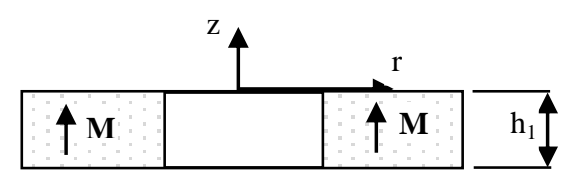

b)

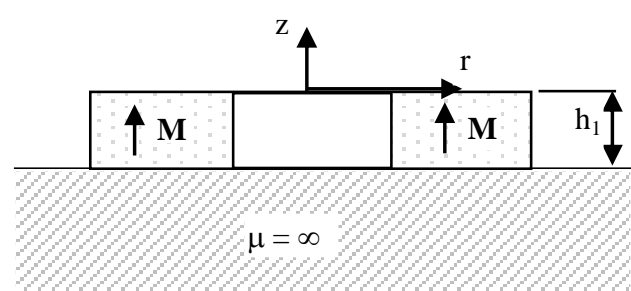

c)
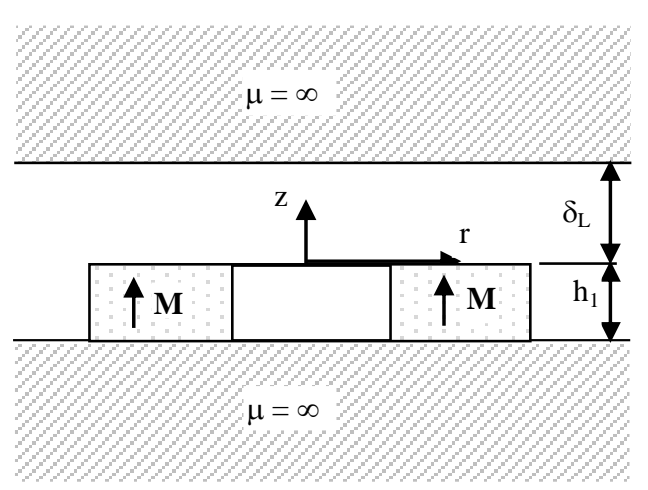

Fig. 3. Magnet Systems:
a) Magnet in air gap region
b) One-sided back iron
c) Double-sided back iron

\section{FORCE CALCULATION}

The force between two magnets can be computed using the basic relation for the force on a distribution of current in an external field in axial direction $\left(B_{z}\right)$

$$
F=\int J(r) \times B_{z}(r) d V
$$

The idea is to represent one of the magnets as a distribution of equivalent currents and then to consider the field due to the other magnet as the external field (which can be computed from eq. (28)).

With eqs. (7) and (8), the force on a magnetized body in the presence of an external field is

$$
F=\int_{V} J_{M} \times B_{z}(r) d V+\int_{S} j_{M} \times B_{z}(r) d S
$$

With eq. (10), (35) reduces to

$$
\boldsymbol{F}=M_{2} \sum_{j^{\prime}=1}^{2}(-1)^{j^{\prime}} \int_{z(1)}^{z(2)} \int_{0}^{2 \pi} \boldsymbol{B}_{\mathbf{z}}(\boldsymbol{r}) \cdot r\left(j^{\prime}\right) d \theta d z
$$

where $M_{2}$ is the magnetization of the magnet 2 as shown in Fig. 1.

Similarity to magnet 1 , there are two surfaces to the magnet 2 to consider

$$
\begin{aligned}
& \text { inner surface }=\left\{\begin{array}{l}
r=R_{21} \\
0 \leq \theta \leq 2 \pi \\
z(1) \leq z \leq z(2)
\end{array}\right. \\
& \text { outer surface }=\left\{\begin{array}{l}
r=R_{22} \\
0 \leq \theta \leq 2 \pi \\
z(1) \leq z \leq z(2)
\end{array}\right.
\end{aligned}
$$

Substitution of (28), (37) and (38) into (36) yields

$$
\begin{aligned}
& F_{z}(z)=\frac{\mu_{o} M_{1} M_{2}}{2} \sum_{j^{\prime}=1}^{2}(-1)^{j^{\prime}} \int_{z(1)}^{z(2)} \int_{0}^{2 \pi}\left[\sum_{j=1}^{2} \sum_{k=1}^{2}(-1)^{(j+k)} \times\right. \\
& \quad \times\left(\frac{1}{N_{\theta}} \sum_{m=0}^{N_{\theta}} \mathrm{S}_{\theta}(\mathrm{m})\left(r \cos \left(\theta-\theta^{\prime}(m)\right)-r^{\prime}(j)\right) r^{\prime}(j) \times\right. \\
& \left.\times I_{1}\left(r\left(j^{\prime}\right), \theta, z ; r^{\prime}(j), \theta^{\prime}(m), z^{\prime}(k)\right)\right] r\left(j^{\prime}\right) d \theta d z
\end{aligned}
$$

Eq. (39) can be simplified with Simpson's method,

$$
\begin{aligned}
& F_{z}(z)=\frac{\mu_{o} M_{1} M_{2}}{2} \sum_{j^{\prime}=1}^{2} \sum_{j=1}^{2} \sum_{k=1}^{2}(-1)^{\left(j+k+j^{\prime}\right)} \times\left(\frac{1}{N_{\theta}^{\prime} N_{\theta}} \times\right. \\
& \sum_{m^{\prime}}^{N_{\theta}^{\prime}} \sum_{m=0}^{N_{\theta}} S_{\theta}^{\prime}\left(m^{\prime}\right) \mathrm{S}_{\theta}(\mathrm{m})\left(r\left(j^{\prime}\right) \cos \left(\theta\left(m^{\prime}\right)-\theta^{\prime}(m)\right)-r^{\prime}(j)\right) r^{\prime}(j) \times \\
& \left.\times r\left(j^{\prime}\right) \int_{z(1)}^{z(2)} I_{1}\left(r\left(j^{\prime}\right), \theta\left(m^{\prime}\right), z ; r^{\prime}(j), \theta^{\prime}(m), z^{\prime}(k)\right)\right) d z
\end{aligned}
$$


The remaining integrations in $\mathrm{z}$ are evaluated with (51) of the Appendix. Use of this result yields

$$
\begin{aligned}
& F_{z}(z)=\frac{\mu_{o} M_{1} M_{2}}{2} \sum_{j^{\prime}=1}^{2} \sum_{k^{\prime}=1}^{2} \sum_{j=1}^{2} \sum_{k=1}^{2}(-1)^{\left(j+k+j^{\prime}+k^{\prime}\right)} \times\left(\frac{1}{N_{\theta}^{\prime} N_{\theta}} \times\right. \\
& \times \sum_{m^{\prime}} \sum_{m=0}^{N_{\theta}^{\prime}} S_{\theta}^{\prime}\left(m^{\prime}\right) S_{\theta}(\mathrm{m})\left(r\left(j^{\prime}\right) \cos \left(\theta\left(m^{\prime}\right)-\theta^{\prime}(m)\right)-r^{\prime}(j)\right) r^{\prime}(j) \times \\
& \left.\times r\left(j^{\prime}\right) \times I_{2}\left(r\left(j^{\prime}\right), \theta\left(m^{\prime}\right), z\left(k^{\prime}\right) ; r^{\prime}(j), \theta^{\prime}(m), z^{\prime}(k)\right)\right) d z
\end{aligned}
$$

where $I_{2}$ is defined in Appendix and the positions $z(1)$ and $z(2)$ are given in the following equation

$z(1)=\delta, z(2)=\delta+h_{2}$

By taking into account the effect of back iron according to Fig. 4, the force can be considered to be a function of the spatial coordinates and the axial positions of the two magnet, i.e.,

$F_{z}\left(z, z^{\prime}\right)=F_{z}\left(z(1), z(2) ; z^{\prime}(1), z^{\prime}(2)\right)$

According to the magnetic circuit shown in Fig. 4a and Fig.4b, the positions $z^{\prime}(1)$ and $z^{\prime}(2)$ are given in (30) and (31), respectively.

While under the influence of double-sided back iron according to Fig.4c, the force equation can be expressed as

$F_{z}\left(z, z^{\prime}\right)=\sum_{i=-\infty}^{+\infty} F_{z}\left(z(1), z(2) ; z^{\prime}(1), z^{\prime}(2)\right)$

where the axial positions are given by the following recursive relations

$z^{\prime}(1)=-2 h_{1}+2 i\left(\delta+h_{1}+h_{2}\right)$ and

$z^{\prime}(2)=2 i\left(\delta+h_{1}+h_{2}\right)$

where $\delta$ is the air gap length between the two magnets.

\section{RESULTS}

The theory was applied to an axially polarized cylinder type $\mathrm{NdFeB}$ magnets for the different magnet systems shown in Figs. 3 and 4 and verified experimentally. The parameters of the magnets used in the analysis are illustrated in Table 1.

Computer programs are developed to calculate the magnetic flux density for the different magnet arrangements. For the field calculation as function of axial position $z$, the magnet is oriented symmetrically with respect to the $x-y$ plane (i.e $\mathrm{z}=0$ corresponds to the middle of the cylinder).
TABLE 1. PARAMETERS OF THE MAGNETS

\begin{tabular}{|l|l|}
\hline \multicolumn{1}{|c|}{ Magnet 1 } & \multicolumn{1}{c|}{ Magnet 2 } \\
\hline$M_{1}=930 \mathrm{kA} / \mathrm{m}$ & $M_{2}=930 \mathrm{kA} / \mathrm{m}$ \\
$R_{11}=6 \mathrm{~mm}$ & $R_{21}=6 \mathrm{~mm}$ \\
$R_{12}=12.5 \mathrm{~mm}$ & $R_{22}=12.5 \mathrm{~mm}$ \\
$h_{l}=16 \mathrm{~mm}$ & $h_{2}=8,16$ or $24 \mathrm{~mm}$ \\
& $\delta=0-16 \mathrm{~mm}$ \\
\hline
\end{tabular}

a)

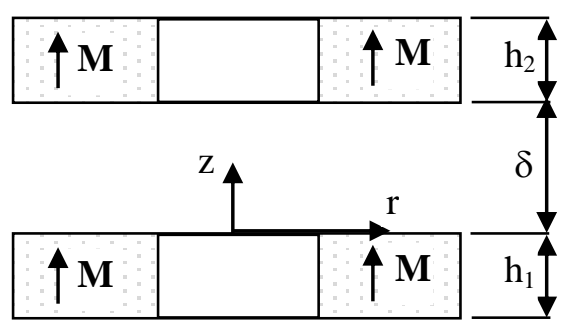

b)

c)
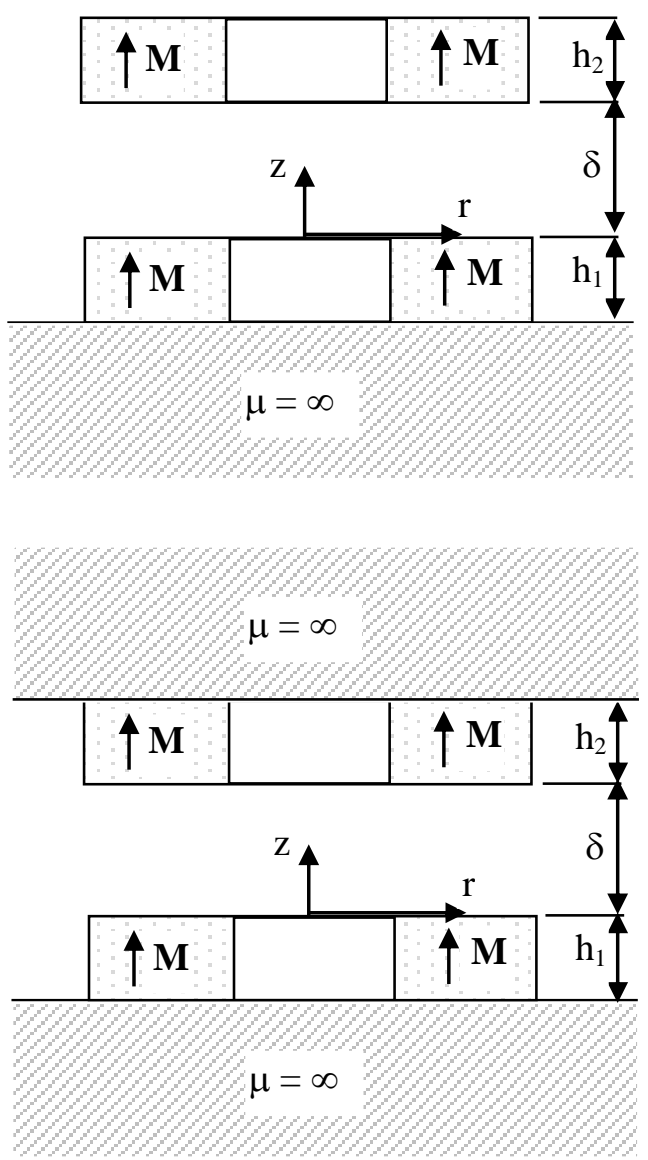

Fig. 4. Magnet Systems for force calculation

a) Magnet in air gap region

b) One-sided back iron

c) Double-sided back iron

The field values of $B_{z}$ were computed for different radial values and different magnet arrangements in Fig. 3. These results are shown in figs. 5 a-c. 


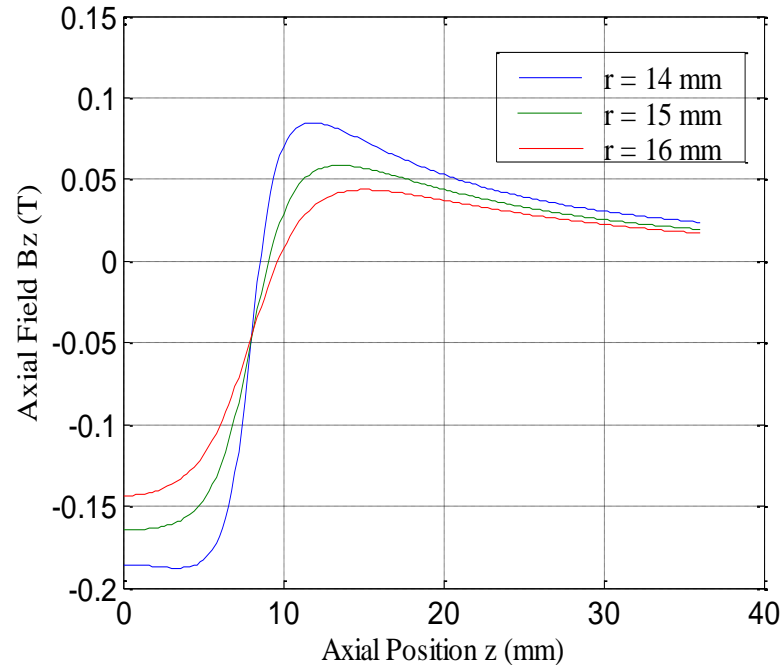

(a)

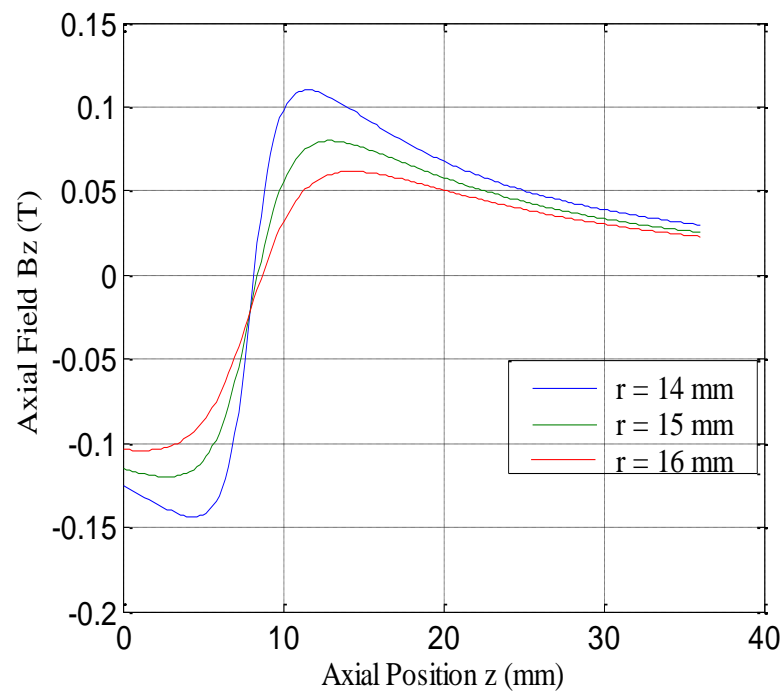

(b)

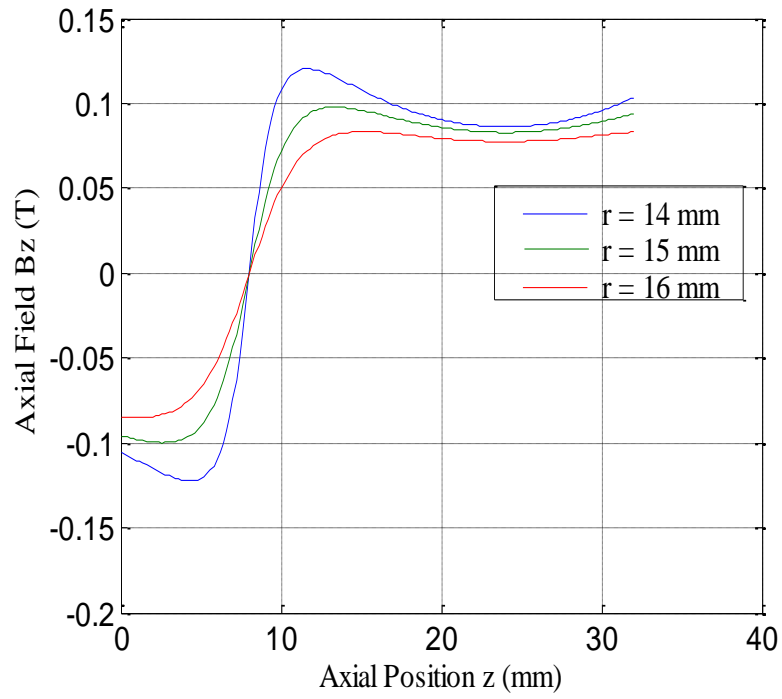

(c)

Fig. 5. Axial field vs. axial position $z$ with radial position $r$ as parameter and $\theta=0$ for the magnet arrangements of Fig. 3:

a) Fig. 3a; b) Fig. 3b; c) Fig. 3c

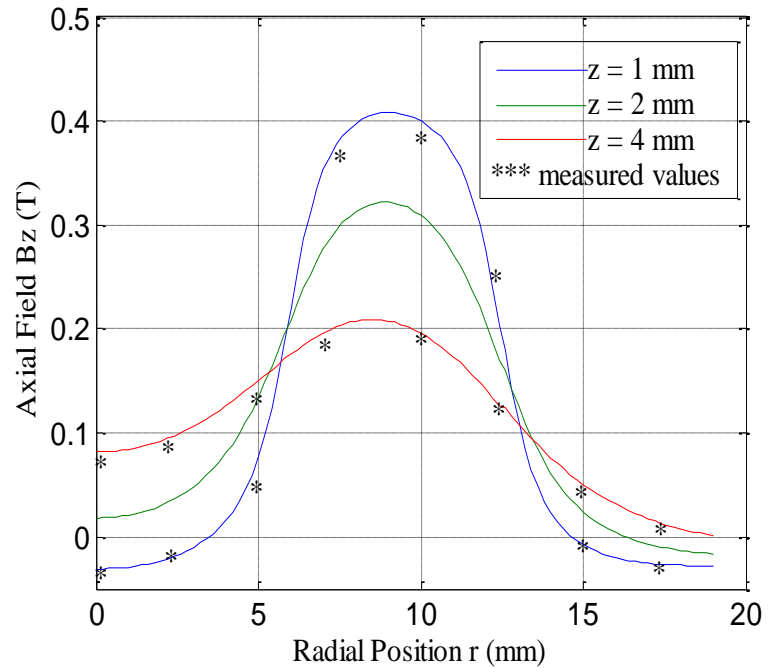

(a)

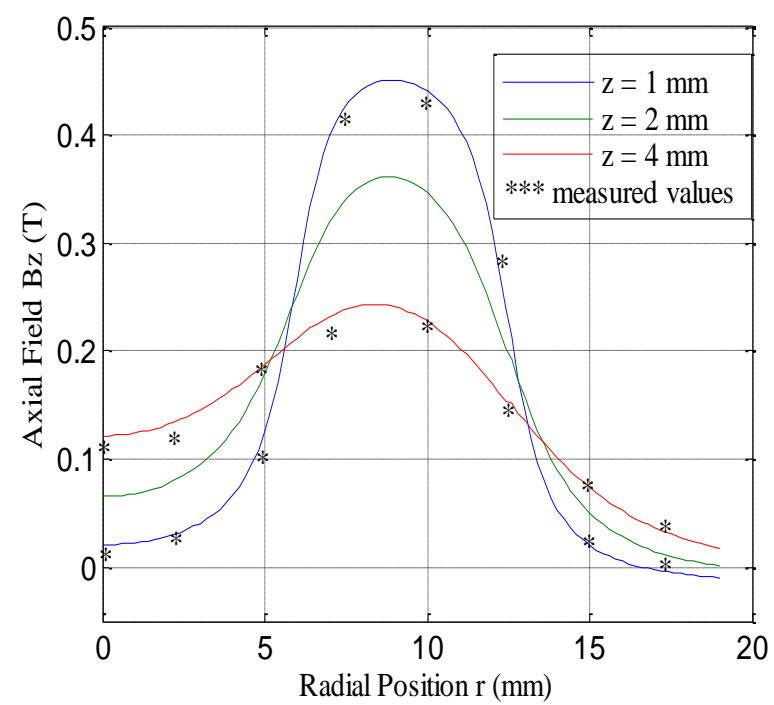

(b)

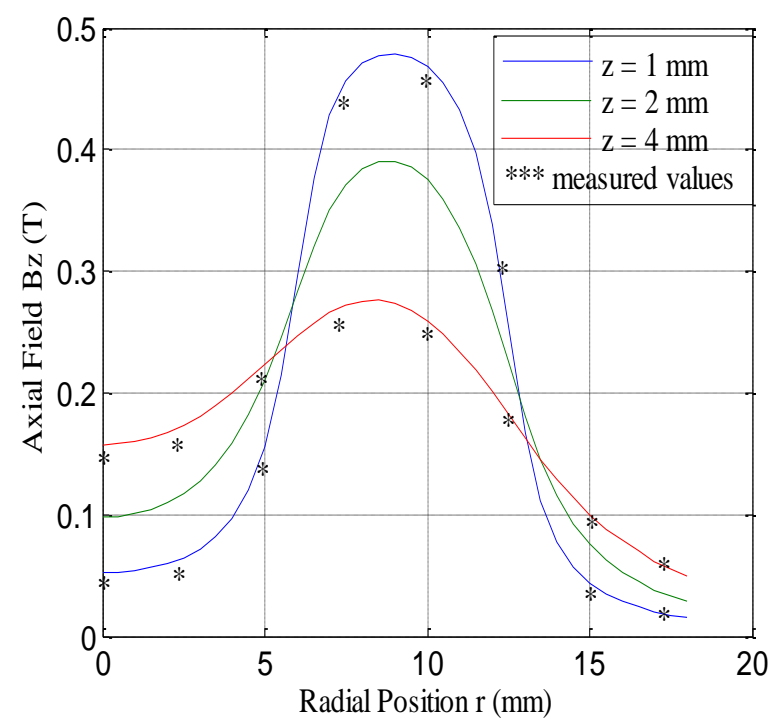

(c)

Fig. 6. Axial field vs. radial position $r$ with axial position $z$ as parameter and $\theta=0$ for the magnet arrangements of Fig. 3:

a)Fig. 3a; b) Fig. 3b; c) Fig. 3c 
For the field calculation of $B_{z}$ as function of radial position $r$, the magnet is oriented with upper surface in $\mathrm{x}-\mathrm{y}$ plane $(z=$ $0)$. The field components $\mathrm{Bz}$ were computed along radial line above the surface of the magnet $(z=1,2$ and $4 \mathrm{~mm}, \theta=$ $0^{\circ}$ ). The data for these calculations appear in figs. $6 \mathrm{a}-\mathrm{c}$ ). The calculated values are compared with experimental results for some curves as shown in fig. 6 a-c) at $z=1$ and 4 $\mathrm{mm}$. The magnetic flux density has been measured by using GAUSS METER Type 3251 (YOKOGAWA) with its probe. In general, the measured values results show good agreement with the theoretical results.

Note that in different cases, the computed field values are slightly higher than the corresponding measured data. The reason for this difference is that the assumption of infinite iron back for some cases does not take into account the fringing (leakage) flux. Nevertheless, the predicted data are sufficiently accurate for parametric design and optimization. The force values were obtained for a series of separation distances which were on the order of a few millimeters and different magnet height $h_{2}$. These values were obtained for different magnet arrangements of Fig. 4 and illustrated in Figs. 7,8 and 9.

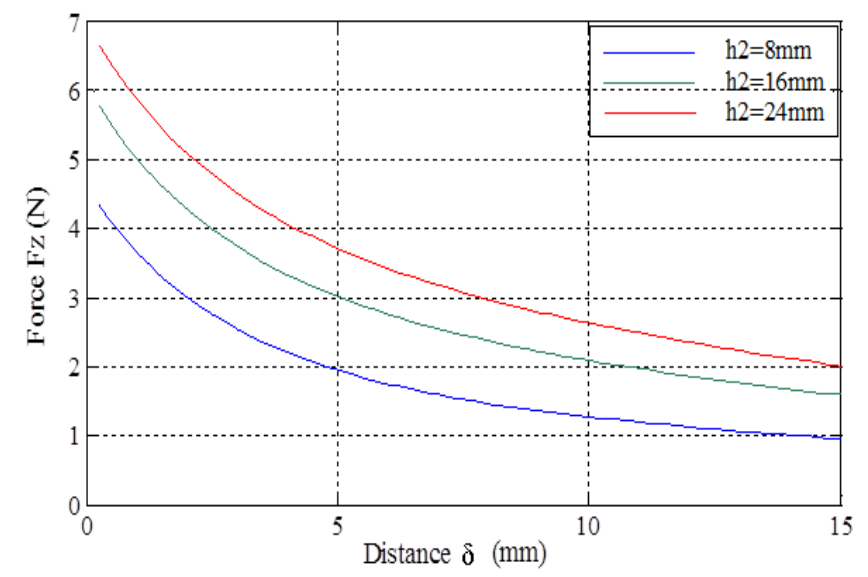

Fig. 7. Magnetic force vs. $\delta$ according to Fig. $4 \mathrm{a}$ with $\mathrm{h}_{2}$ as parameter

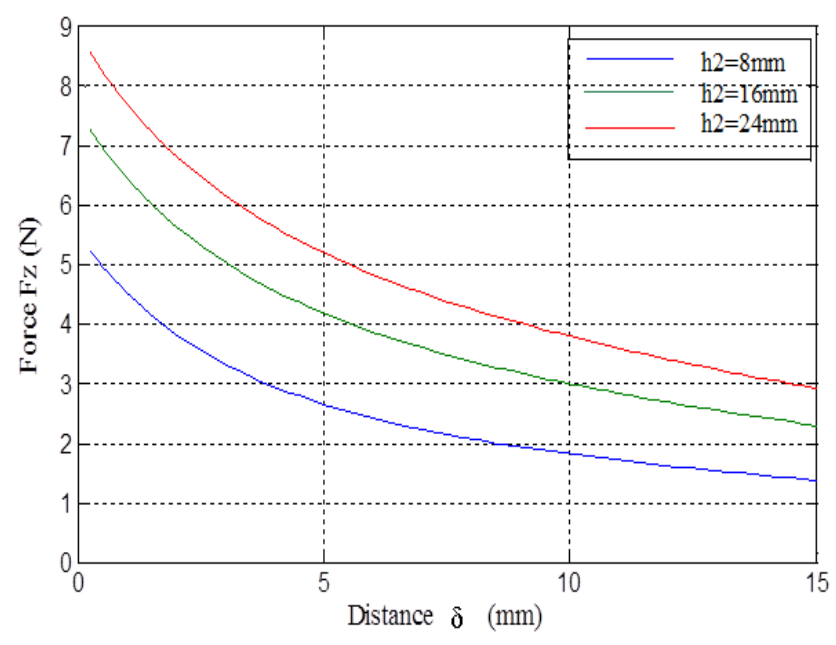

Fig. 8. Magnetic force vs. $\delta$ according to Fig. $4 \mathrm{~b}$ with $\mathrm{h}_{2}$ as parameter

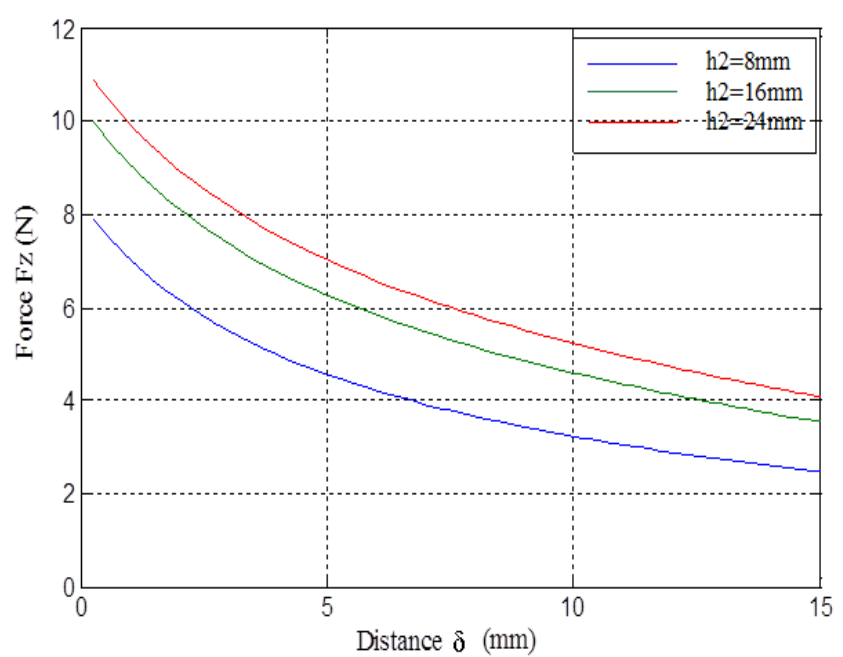

Fig. 9. Magnetic force vs. $\delta$ according to Fig. $4 \mathrm{c}$ with $\mathrm{h}_{2}$ as parameter

It is important to note that when programming the multipole summations in field and force equations, a higher degree of accuracy can be achieved with fewer mesh points if the total of a single summation over one index is evaluated and saved separately (as an intermediate step), and then the totals of the successive summations are added together to obtain the final sum.

\section{CONCLUSION}

A three dimensional field solution has been derived for axially polarized magnetic cylinder for different magnet arrangements. The calculation is based on the assumption of ideal magnetization and is applicable to rare earth material such as $\mathrm{NdFeB}$. The expressions for the field components have been expressed in terms of elementary functions that are readily programmed. Moreover, they allow rapid parametric studies of field strength and could be of considerable use in the design and optimization of numerous devices. The computation results for the flux density are in good agreement with the experimental data for different magnet arrangements.

\section{APPENDIX}

In this section, the variables $\theta^{\prime}(m), S_{\theta}(m)$ and $I_{1}$ that appear in (27) and (28) and the variables $\left.\theta\left(m^{\prime}\right), S^{\prime} d^{\prime} m^{\prime}\right)$ and $I_{2}$ that appear in (40) and (41) are defined. Specifically

$$
\begin{aligned}
& \theta^{\prime}(m)=2 \pi m / N_{\theta} \\
& \theta\left(m^{\prime}\right)=2 \pi m^{\prime} / N_{\theta}^{\prime} \\
& S_{\theta}(m)= \begin{cases}1 / 3 & (m=0) \\
4 / 3 & (m=1,3,5, \ldots) \\
2 / 3 & (m=2,4,6, \ldots) \\
1 / 3 & \left(m=N_{\theta}\right)\end{cases}
\end{aligned}
$$


and

$$
S_{\theta}^{\prime}\left(m^{\prime}\right)= \begin{cases}1 / 3 & \left(m^{\prime}=0\right) \\ 4 / 3 & \left(m^{\prime}=1,3,5, \ldots\right) \\ 2 / 3 & \left(m^{\prime}=2,4,6, \ldots\right) \\ 1 / 3 & \left(m^{\prime}=N_{\theta}^{\prime}\right)\end{cases}
$$

Lastly

$$
I_{1}\left(r, \theta, z ; r(j), \theta^{\prime}(m), z(k)\right)=
$$

$=\left\{\begin{array}{l}\frac{(z-z(k)) G\left(r, \theta, z ; r(j), \theta^{\prime}(m), z(k)\right)}{r^{2}+r^{2}(j)-2 r r(j) \cos \left(\theta-\theta^{\prime}(m)\right)} \\ \text { for }\left(r^{2}+r^{2}(j)-2 r r(j) \cos \left(\theta-\theta^{\prime}(m)\right) \neq 0\right) \\ \frac{-1}{2(z-z(k))^{2}} \\ \text { for }\left(\cos \left(\theta-\theta^{\prime}(m)\right)=1, r=r(j), z \neq z(k)\right)\end{array}\right.$

and

$$
I_{2}\left(r\left(j^{\prime}\right), \theta\left(m^{\prime}\right), z\left(k^{\prime}\right) ; r^{\prime}(j), \theta^{\prime}(m), z^{\prime}(k)\right)=
$$

$$
=\left\{\begin{array}{l}
\frac{G^{-1}\left(r\left(j^{\prime}\right), \theta\left(m^{\prime}\right), z\left(k^{\prime}\right) ; r^{\prime}(j), \theta^{\prime}(m), z^{\prime}(k)\right)}{r^{2}\left(j^{\prime}\right)+r^{\prime 2}(j)-2 r\left(j^{\prime}\right) r^{\prime}(j) \cos \left(\theta\left(m^{\prime}\right)-\theta^{\prime}(m)\right)} \\
\text { for }\left(r^{2}\left(j^{\prime}\right)+r^{\prime 2}(j)-2 r\left(j^{\prime}\right) r^{\prime}(j) \cos \left(\theta\left(m^{\prime}\right)-\theta^{\prime}(m)\right) \neq 0\right) \\
\frac{1}{2\left(z\left(k^{\prime}\right)-z^{\prime}(k)\right)} \\
\text { for }\left(\cos \left(\theta\left(m^{\prime}\right)-\theta^{\prime}(m)\right)=1, r\left(j^{\prime}\right)=r^{\prime}(j), z\left(k^{\prime}\right) \neq z^{\prime}(k)\right)
\end{array}\right.
$$

\section{REFERENCES}

[1] E. P. Furlani, A three-dimensional field solution for axially polarized multipole disks, J. Magn. Magn. Mater., vol. 135, pp. 205-214, 1994.

[2] E. P. Furlani, A three-dimensional field solution for permanentmagnet axial-field motors, IEEE Trans. Magn., vol. 33, no. 3, pp. 2322-2325, May 1997.

[3] Y. N. Zhilichev, Calculation of 3D magnetic field of disk-type micromotors by integral transformation method, IEEE Trans. Magn., vol. 32, no. 1, pp. 248-253, January 1996.

[4] D. K. Cheng, Field and wave electromagnetic, Addison-Wesley Publishing Company, 1989.

[5] Y. N. Zhilichev, Three-dimensional analytic model of permanent magnet axial flux machine, IEEE Trans. Magn., vol. 34, no. 6, pp. 3897-3901, November 1998.

[6] D. A. González, Tapia, J. A. and Bettancount, A. L., Design consideration to reduce cogging torque in axial flux permanentmachines, IEEE Trans. Magn., vol. 43, no. 8, pp. 3435-3439, August 2007.

[7] J. Azzouzi, G. Barakat, and B. Dakyo, Quasi-3-D analytical modeling of the magnetic field of an axial flux permanent-magnet synchronous machine, IEEE Trans. Energy Convers., vol. 20, no. 4, pp. 746-752, December 2005.

[8] T.J.E. Miller, Brushless permanent-magnet and reluctance motor drives, Clarendon Press, Oxford, 1989.
[9] H. B. Ertan, M. Y. Üctug, R. Colyer, and A. Consoli, Modern electrical drives, Kluwer Academic Publishers, 2000.

[10] A.-K. Daud, , R. Hanitsch, A three-dimensional field solution for axially-polarized permanent-magnet cylinders for different magnetic arrangements, Electromotion, January-March 2008, Issue 1 ,Volume 15 , pp. $3-12$

\section{Creative Commons Attribution License 4.0} (Attribution 4.0 International, CC BY 4.0)

This article is published under the terms of the Creative Commons Attribution License 4.0 https://creativecommons.org/licenses/by/4.0/deed.en_US 